\title{
Calculation and optimization of OSNR based on long-distance OTN network
}

\author{
SUN Yan-zhi ${ }^{1, a}$, HU Jin-song ${ }^{1, b}$, LIU Yu-ming ${ }^{1, c}$, TIAN Zhi-jun ${ }^{1, d}$ and CHAI \\ Hui-yi ${ }^{2, e}$ \\ ${ }^{1}$ China Southern Power Grid Co., Ltd, China \\ ${ }^{2}$ Institute of Information Photonics and Optical Communications, Beijing University of Posts and \\ Telecommunications, China

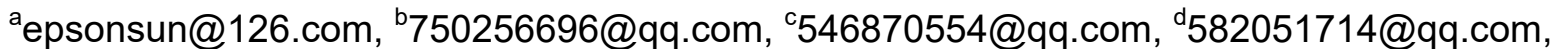 \\ echaihuiyi1992@bupt.edu.cn
}

Keywords: OSNR, OTN, calculation and optimization

\begin{abstract}
With the rapid development of national power grid, large-scale electric power communication network is evolving into OTN network in order to back up the power grid management, operation and maintenance of a large number of data communication. Long-distance optical communication is the characteristic of large-scale OTN network, as a result, the calculation of OSNR plays a critical role in research and application to the network. This paper will focus on the analysis of the topology characteristics of long-distance OTN power communication network. The OSNR calculation method based on the topology structure will be proposed, and the features, significance and popularization value of this method will be analyzed.
\end{abstract}

\section{Introduction}

With the rapid development of economy, the continuous progress of society, the level of science and technology and the level of information technology improved continuously, the demand for electricity is increasing, and the size of the power grid expand continuously too. In order to meet the needs of the current electricity demand and analyze the development trend of power grid, we will use all optical cross and 10 gigabit interconnection of OTN technology in the next step to support a large number of data generated from network. In order to ensure the connection of the optical path and the performance of the whole network, we must calculate and optimize the optical signal noise ratio (OSNR), which is the most important parameter in the physical layer of the long distance OTN network. Good OSNR parameter is the basis of wavelength division multiplexing system operation, and is also one of the important factors to be considered in the network planning and upgrading. This paper will improve the communication efficiency of OTN network, and guarantee the user's services to meet the needs of the more open market environment through the research of the calculation of OSNR.

\section{OSNR}

Definition. OSNR (Optical Signal Noise Ratio). The optical signal noise ratio is defined as the ratio of the optical signal power and the noise power in the optical effective bandwidth is $0.1 \mathrm{~nm}$. [1] The power of the optical signal is generally taken peak, and the power of the noise is generally taken as the intermediate point of the two adjacent channels.

OSNR Influencing factors. For the transmission of information in WDM systems, so far, the main factor to limit the transmission distance is: optical signal noise ratio (OSNR), dispersion and nonlinear. [2] For dispersion, can be solved by the dispersion compensation fiber. The optical signal noise ratio (OSNR) can be solved by the Raman amplifier and super FEC technology. 


\section{OSNR Calculation}

Optical signal noise ratio is defined as the ratio of the optical signal power and the noise power. OSNR as an important indicator of WDM/OTN, optical signal noise ratio's calculation is very complicated in different systems, the calculation methods are quite different.

At present, there are many different calculation methods, based on different conditions, roughly divided into the following categories.

When the value of power is known or can be measured by physical measurement. According to the definition of optical signal noise ratio, it can be calculated that use the signal power received by the receiving end of the optical signal divided by the power of noise within the $0.1 \mathrm{~nm}$ bandwidth, the result are expressed in the form of $\mathrm{dBm}$.

When the system is composed of a number of the same EDFA. In the G.692 ITU-T file, if the all the EDFA connected by optical link in the system has the same noise index (F), gain and the attenuation value(L) of each section of the optical fiber are the same, the optical signal noise ratio can be calculated as [3]

$$
R_{\text {out }}=F_{\text {tn }}-F-10 \mathrm{gg} N-10 \lg \left(\mathrm{hv} B_{0}\right)
$$

When the the attenuation of optical fiber which connected amplifier are the same, and the gain of the amplifier just enough compensate the attenuation. Assuming that the total output power of each amplifier is equal, and the amplifier gain is greater than 1, then the OSNR on the output side can be expressed as [4]

$$
\text { OSNR }=P_{\text {OUT }}-L-N_{F}-10 \lg N-10 \lg \left[h v \Delta B_{0}\right]
$$

When attenuation value of the fiber link is much smaller than the noise index of the amplifier. The attenuation of optical transmission section in the multiplexing system and length are not exactly the same, and optical amplifier which in different system, the configurations are not exactly the same, after simplified, OSNR will be calculated as [5]

$$
\mathrm{R}_{K}=58-1018 \sum_{j=1}^{z} 10^{\frac{E_{j}-E_{j}}{10}}
$$

Summary of different OSNR calculation methods. The calculation method of OSNR is usually divided into the categories of measurement, definition and calculation.

Measurement is getting the value of OSNR in the system by using the optical signal spectrum measured by the spectrum analyzer. In this process, different measurement techniques and principles can be used to obtain the corresponding OSNR by using different calculation method. For example, the principle of interpolation method, signal shutdown method, polarization analysis method.The definition is mainly based on the definition of the optical signal noise ratio itself, get the power of the signal and noise to calculate directly, and then calculate the value of the optical signal noise ratio.Calculation is mainly based on the principle of noise generation, the principle of noise generation will be different according to the different systems. Different calculation methods are based on certain assumptions of the fixed system, so the calculation methods are different.

\section{OSNR calculation based on long distance OTN network}

Development status of OTN network. With the development of broadband data service and OTN technology, it is necessary to build a more efficient and reliable transmission network based on OTN technology. OTN technology, as a new optical network transmission technology, inherits and develops the advantages of the existing transmission network, is one of the best transmission technology for broadband customer data business. The application of OTN technology, the choice of OTN equipment type, the relationship between the IP layer and the OTN layer and the relationship between the OTN network and other network are the key problems in the application of OTN technology.

Analysis of long-distance OTN network. According to the fact, long-distance OTN network has the following characteristics: 
1) Attenuation of the different sections of optical fiber are various, cannot calculated only by using optical fiber attenuation coefficient;

2) The value of optical fiber attenuation cannot be negligible by comparing to the amplifier gain;

3) Cannot get the noise power at the output by measure directly;

4) The total power on the output can be measured.

Therefore, based on the above characteristics, we need to consider is that in this type of system, the noise accumulation has greater impact on the OSNR, OSNR calculation needs analysis from the system:

The thumbnail of specify optical amplifier transmission system is shown in Figure 1, the optical amplifier in the figure are OTM terminal, the gain range of OTM terminal and the loss of different sections of the optical fiber are various. Where $G_{i}(d B)$ represents the gain of the $i$-th OTM terminal, $L_{i}$ represents the loss $(d B)$ of the $i$-th optical fiber.

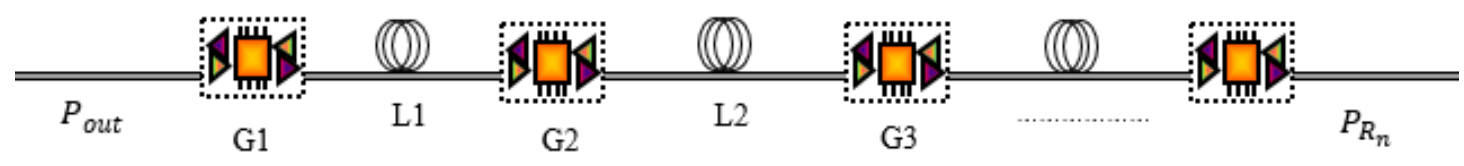

Fig. 1 OTM terminal cascade system transmission network structure

In the form of $\mathrm{dBm}$, analysis the optical signal noise ratio at $\mathrm{R}_{\mathrm{n}}$ into the following steps:

1) Calculate the ASE noise power generated by each OTM terminal:

$F_{i}=N F_{i}+G_{i}+10 \lg \left(M D_{0}\right)$

Where $\mathrm{NF}_{\mathrm{i}}(\mathrm{dB})$ is the noise coefficient of the $\mathrm{i}$-th optical amplifier;

2) Calculate the optical signal noise ratio of the $R_{n}$ point:

$\operatorname{OSNR}(\mathrm{dB})=P_{\text {OUT }}-\sum_{k=1}^{j}\left(\mathrm{P}_{\mathrm{k}}-\sum_{m=k}^{j-1} L_{m}+\sum_{n=k+1}^{j} G_{n}\right)$

Where: $\mathrm{P}_{\text {out }}$ is power of sender transmit into the fiber, $\mathrm{P}_{\mathrm{k}}$ is the noise power generated by OTM terminal, sender send signal amplification and attenuation through $\mathrm{j}$ OTM terminals and reach the receiver. Since the fiber attenuation value cannot be negligible compared to the amplifier gain, the noise generated by each OTM terminal will be amplified by the next amplifier and attenuated by the fiber link. The noise power received at the receiver is summed up by the noise of each amplifier send through the link to the receiver. According to the definition of OSNR, the value of OSNR can be obtained by subtracting the noise power from the power of the signal.

The application and popularization of OSNR calculation. In this article, OSNR calculation methods are analyzed and compared based on the different system, combined with the actual situation of long distance OTN network, a new OSNR calculation method is been proposed. It is very meaningful to apply and promote the calculation method proposed in the paper.

Based on the actual situation and the actual topology, the formulas and calculation method proposed in this paper is suitable for most of OTN network. The method to calculate the OSNR can be used in the OTN network which has the following characteristics:

1) Has the basic characteristics and performance of long-distance OTN network;

2) All fiber amplifier equipment are connected by the optical fiber;

3) There is a certain physical distance between all optical amplifiers;

4) Noise coefficient and gain of optical amplifier equipment and the fiber loss are known;

5) The signal total power received from the last optical amplifier is measurable.

Based on the characteristics of OTN network, Compared with the first four OSNR calculation methods, the calculation method of the OSNR proposed in this paper, which has more extensively applicable scope and it needs less calculation steps, and easy calculation process.

OSNR calculation results. The calculation method based on analyzing the characteristics of the network needs to be verified by practical. According to the actual situation of national power grid at present, the calculation method of the OSNR for long distance OTN networks is applicable to a more consummate network system in this paper. The network itself must built within a city or between 
cities. We select a province actual situation to analyze the actual topology. The actual situation of the province's network is in accordance with the characteristics of long distance OTN network and requirements:

This province OTN network covers more than ten cities, more than sixty sites. This province integrated data network set two data centers as the core node, 10 Gigabit bandwidth interconnection, and backup each other. Each city is open two 10GE WDM service sub-channel to confluence to the core nodes. Overall, the occupancy rate of province OTN network link is more abundant, it can met the new business access needs in these years.

According to the actual situation of the province, extract representative lines made by routing and alternate routing as the practical application.

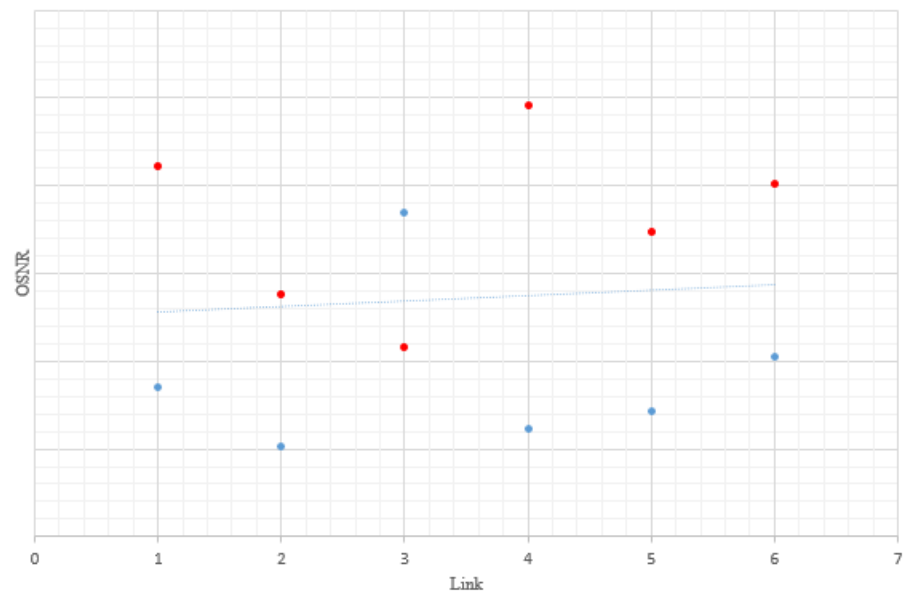

Fig. 2 Calculation result

The analysis results through this graph are as follows:

1) The value of the OSNR in the system are in reasonable range;

2) The value of OSNR of routing and alternate routing in the system are similar;

3) The value of OSNR of most of the route in the system are within the range.

\section{Conclusion}

Nowadays, based on more and more studies of OSNR, there are many calculation methods of OSNR. But these calculation methods are based on assumptions in different systems, so the scheme of OSNR calculation generally have some limitations .In this paper, the solution is more closed to the actual situation of system, and the applicability is more widely, so in this area there is still much room for development.

\section{References}

[1] Information on http://baike.baidu.com/link?url=brcuCUGKNPm37rTGtY3R9QeQ0FpDf0DKLStsbD31qOOOsYsv LJXYbot3HALnzoAEKUV4Pr72enRpDxpw6Nayt_

[2] Liu Haitao, Zhan Tao, Yang Bin, Telecommunication transmission [J]. Post and Telecommunications Design Technology, 2013, 3:p. 58-61

[3] ITU-T Rec G.692[S].

[4] Zheng Bo, Telecommunication transmission [J]. Post and Telecommunications Design Technology, 2014, 1(1):p. 60-66

[5] Liu Haitao, Zhan Tao, Yang Bin, Telecommunication transmission [J]. Post and Telecommunications Design Technology, 2013, 3:p. 58-61 